\title{
No clinical relevance of approved animal experiments after seventeen years
}

In 2005 we published an assessment of the medical impact of 17 research projects involving animal experiments and their 65 resulting primary publications (Lindl et al., 2005). Here we extend the original review, which covered the years 1993-2004, to include the period from 2005 to March 2011.

This investigation included all proposals for animal experiments from the universities of Wuerzburg, Erlangen, and Regensburg - as well as proposals from drug companies in northern Bavaria - that were approved by the Ethical Commission on Animal Experimentation in Wuerzburg, Germany between 1991 and 1993. Of the 51 proposals, 17 (33\%) were retrospectively considered successful within the animal models on the basis of their aims stated in the written proposals (Lindl et al., 2001). These projects resulted in 65 primary publications. Citations of the primary publications were compiled using the aid of such international data banks as PubMed, DIMDI, and other science-oriented data retrieval systems, including MedLine and EMBASE.

We found 1,316 citations of the 42 primary publications arising from the 10 basic research proposals and 146 citations of the 23 primary publications arising from the seven applied research proposals. More than half the citations in each field were made in papers reporting other in vivo animal experiments. The citation maximum for basic research papers was observed in 1998 (129); for applied research papers in 1996 (29). The decline in citations of the original animal data to zero in 2010 exemplifies the lack of long-term impact of most animal experiments.

Of the citations to publications in the field of applied research, 132 were found in clinically oriented papers, of which only seven related the experimental animal data to therapeutic results in humans. Even these seven reviews or review-like publications did not provide evidence of a direct relationship between the data from animal experiments and human therapies. In some instances, the animal data did not predict the human response.

Our data are in line with other investigations that followed up animal experiments (Reines, 1991; Plous and Herzog, 2001; Grant et al., 2003; Dagg and Seidle, 2004; Pound et. al., 2004; Knight, 2007, 2008), and also concluded that the clinical benefits of animal experiments for humans are overestimated. Reasons for this may lie in the species difference (Pound et al., 2004) and/or in poor design, standardization, and statistical power of animal experiments (Faggion et al., 2009; Gruber and Hartung, 2004; Balls, 2010). This mounting evidence seriously undermines the dogma that animal experiments are indispensable for clinical research progress.

We recommend that applications to perform animal experiments must consider the species difference and include a detailed argument on why the experiments are indispensable. Authorities should have the right to require alteration of the proposals, e.g. regarding the study design, animal numbers, etc., before approval (Foster and Braddock, 2004). Harmful experiments on animals must be restricted more efficiently and be weighed more carefully against the potential gain of knowledge in light of this type of retrospective analysis (Lindl et al., 2005; Dagg and Seidle, 2004).

Our results, although obtained within a restricted geographical area, should be a cause for reflection, both for researchers and for the authorities responsible for licensing animal experiments.

\section{References}

Balls, M. (2010). The principles of humane experimental technique: Timeless insights and unheeded warnings. ALTEX 27, 144-148.

Dagg, A. I., and Seidle, T. K. (2004). Levels of citation of nonhuman animal studies conducted at a Canadian research hospital. J. Appl. Anim. Welf. Sci. 7, 205-213.

Faggion, C. M. Jr, Schmitter, M., and Tu, Y. K. (2009). Assessment of replication of research evidence from animals to humans in studies on peri-implantitis therapy. J. Dent. 37, 737747.

Foster, M., and Braddock, M. (2004). To guide the process. Part II: Animal models. Screening 3, 20-21.

Grant, J., Green, L., and Mason, B. (2003). From bedside to bench: Comroe and Dripps revisited. London: Brund University (HERG Research Report No. 30). 
Gruber, F. P., and Hartung, T. (2004). Alternatives to animal experimentation in basic research. ALTEX 21, Suppl., 3-31.

Knight, A. (2007). Systematic reviews of animal experiments demonstrate poor human clinical and toxicological utility. ATLA 35, 641-659.

Knight, A. (2008). Non animal methodologies within biomedical research and toxicity testing. ALTEX 25, 213-231.

Lindl, T., Weichenmeier, I., Labahn, D., et al. (2001). Evaluation von beantragten und genehmigten tierexperimentellen Versuchsvorhaben in Bezug auf das Forschungsziel, den wissenschaftlichen Nutzen und die medizinische Relevanz (Evaluation of authorised experiments on laboratory animals with regard to the aim of the research to be carried out, its scientific usefulness and its medical relevance). ALTEX 18, 171-178.

Lindl, T., Voelkel, M., and Kolar, R. (2005). Tierversuche in der biomedizinischen Forschung. Eine Bestandsaufnahme der klinischen Relevanz von genehmigten Tierversuchsvorhaben [Animal experiments in biomedical research. An evaluation of the clinical relevance of approved animal experimental projects]. ALTEX 22, 143-151.

Plous, S., and Herzog, H. (2001). Animal research. Reliability of protocol reviews for animal research. Science 293, 608609.
Pound, P., Ebrahim, S., Sandercock, P., et al. (2004). Where is the evidence that animal research benefits humans? BMJ 328 , 514-517.

Reines, B. P. (1991). On the locus of medical discovery. J. Med. Philos. 16, 183-209.

Rieser, T. M. (2005). Urinary tract emergencies. Vet. Clin. North Am. Small. Anim. Pract. 35, 359-373.

Toni Lindl ${ }^{1 *}$ and Manfred Voelkel ${ }^{2}$
${ }^{1}$ Institute of Applied Cell Culture,
Munich, Germany;
${ }^{2}$ Ethical Commission on Animal
Experimentation,
Wuerzburg, Germany
*Corresponding author:
Toni Lindl, PhD
Institut für angewandte Zellkultur
Balanstr. 6,81669 München
Germany
e-mail: I-A-Z@t-online.de

\section{SATIS ethics ranking of universities in Germany regarding animal use in education}

SATIS (latin: enough!) - the humane education project of People for Animal Rights Germany (PARG) - performed the first of its kind ethics ranking to assess the use of animals and need for alternatives in education and to create a guideline for highschool graduates and students, which is accessible at www.satistierrechte.de. We used a questionnaire-based telephone survey and called the responsible teachers for each course related to animal use at each faculty for biology, medicine and veterinary medicine in Germany to ask which animals or alternatives are used in practical classes and whether students have the possibility of conscientious objection. A university ranking was then established by means of specific ethical criteria.

Students of biology, medicine and veterinary medicine are often confronted with the use of animals for dissection or in physiological experiments. While future veterinarians and some biologists need hands on experience with animals, numerous high quality alternatives are available to avoid the harmful use of animals in education. These include models and mannequins, plastinated animals and animal body donation programs. A wide variety of alternatives is already established and evaluated in zoological institutes all over the world (see www.interniche.org; www.humanelearning.info). In this study, we were interested in the use of animals and alternatives at German universities.

The detailed questionnaire for teachers of zoological institutes asked which and how many animals are used in different courses, which alternatives are established and if not, whether students have the right to conscientious objection. Additional questions included the goals of the course using animals or alternatives, the fate of surviving animals after the course and whether there was interest in implementing alternatives. The four-page questionnaire was to be either be filled in by us during a telephone interview with the responsible teacher or sent to the teacher as a writeable pdf file by email. Contact details of responsible teachers and basic information on degrees and curricula were found in a systematic web search.

We contacted the responsible instructors of all 5 faculties for veterinary medicine, 35 medical faculties and 70 biological faculties in Germany. No teacher was ready to perform a detailed telephone interview because of time constraints or lack of interest. While one third of the teachers ordered the writable questionnaire, only four filled it in and returned it. Most information was gathered in shortened telephone calls concentrated on the 